\title{
artigo
}

Lima, B.S.; Aguiar, J.C.; Aguiar, L.M.N.M.; Machado da Silva, D.M.; Carvalho Junior, A.M.; Lima da Silva, L.F.; Matos, R.R.C.

A presença de parasitas em alfaces (Lactuca Sativa) comercializados em feiras no município de Santarém-PA

\section{A presença de parasitas em alfaces (Lactuca Sativa) comercializados em feiras no município de Santarém-PA}

\author{
The presence of lettuce (Lactuca Sativa) parasites commercialized at fairs in Santarém-PA
}

La presencia de parasitos em lechuga (Lactuca Sativa) comercializada em férias em el municipio de Santarém-PA

\begin{abstract}
RESUMO
OBJETIVOS: Avaliar a possível presença de parasitas em amostras de alfaces comercializados em feiras do município de Santarém-PA no ano de 2019. MATERIAL E MÉTODOS: Trata-se de um estudo exploratório, transversal, de método descritivo, e abordagem quanti-qualitativa, onde foram colhidas, entre os meses de agosto a setembro de 2019, 51 amostras da Lactuca sativa, em três feiras de comercialização no município de Santarém-PA. As amostras foram analisadas em laboratório. RESULTADOS: Dentre as amostras analisadas, 16 apresentaram-se negativas para algum parasita e 35 amostras apresentaram-se positivas das quais 07 amostras estavam contaminadas com Balantidium coli e 28 com Giardia Lamblia. CONCLUSÃO: As alfaces analisadas mostraram-se ser um importante meio de transmissão de doenças parasitárias, tendo a necessidade de medidas que melhorem a qualidade higiênica-sanitária das hortaliças.
\end{abstract}

DESCRITORES: Alface; Saúde Pública; Hortaliças; Parasitas.

\section{ABSTRACT}

OBJECTIVES: To evaluate the possible presence of parasites in samples of lettuce sold at fairs in the municipality of Santarém-PA in 2019. MATERIAL AND METHODS: This is an exploratory, cross-sectional study, with a descriptive method, and a quantitative and qualitative approach, where 51 samples of Lactuca sativa were collected between August and September 2019, in three trade fairs in the municipality of Santarém-PA. The samples were analyzed in the laboratory. RESULTS: Among the analyzed samples, 16 were negative for some parasite and 35 samples were positive, of which 07 samples were contaminated with Balantidium coli and 28 with Giardia Lamblia. CONCLUSION: The lettuces analyzed proved to be an important means of transmitting parasitic diseases, with the need for measures that improve the hygienic-sanitary quality of vegetables.

DESCRIPTORS: Lettuce; Public Health; Vegetables; Parasites.

\section{RESUMEN}

OBJETIVOS: Evaluar la posible presencia de parásitos en muestras de lechuga vendidas en ferias en el municipio de Santarém-PA en 2019. MATERIAL Y MÉTODOS: Este es un estudio exploratorio, transversal, con un método descriptivo, y un enfoque cuantitativo y cualitativo, donde se recolectaron 51 muestras de Lactuca sativa entre agosto y septiembre de 2019, en tres ferias en el municipio de Santarém-PA. Las muestras fueron analizadas en el laboratorio. RESULTADOS: Entre las muestras analizadas, 16 fueron negativas para algunos parásitos y 35 muestras positivas, de las cuales 07 fueron contaminadas con BaIantidium coli y 28 con Giardia Lamblia. CONCLUSIÓN: Las lechugas analizadas demostraron ser un medio importante de transmisión de enfermedades parasitarias, con la necesidad de medidas que mejoren la calidad higiénico-sanitaria de los vegetales. DESCRIPTORES: Lechuga; Salud Pública; Verduras; Parásitos.

RECEBIDO EM: 19/02/2020 APROVADO EM: 20/02/2020

\section{Beatriz Silva Lima}

Graduada em Biomedicina do Centro Universitário da Amazônia (UNAMA). https://orcid.org/0000-0001-5481-9693

\section{Jocireudo Carneiro Aguiar}

Mestre em Genética e Biologia Molecular. Docente no Centro Universitário da Amazônia (UNAMA). https://orcid.org/00000002-0706-2883 


\section{Lília Maria Nobre Mendonça de Aguiar}

Mestre em Engenharia de Processos pela Universidade Federal do Pará (UFPA); Especialista em Oncologia; Graduada em Enfermagem; Docência e Preceptora em Ensino Superior; Preceptoria em Ensino Superior. https://orcid.org/0000-0003-27862391

\section{Domingas Machado da Silva}

Enfermeira Mestranda em Biociências pela Universidade Federal do Oeste do Pará (UFOPA). Especialista em Urgência e Emergência (IESPES), Gestão em Saúde (FIOCRUZ) e Saúde Coletiva com Ênfase na Atenção Básica (UNAMA). https://orcid. org/0000-0002-8619-1332

\section{Antenor Matos de Carvalho Junior}

Enfermeiro. Mestrando em Engenharia de Processos pela Universidade Federal do Pará (UFPA). Especialista em Saúde Pública. Docente e Preceptor na Universidade do Estado do Pará (UEPA). https://orcid.org/0000-0001-6368-8939

\section{Lulucha de Fátima Lima da Silva}

Enfermeira. Pós-graduada em Programa de Saúde da Família. Docente no Centro Universitário da Amazônia (UNAMA). https:// orcid.org/0000-0003-1384-6040

\section{Rodrigo Ruan Costa de Matos}

Acadêmico de Medicina pela Universidade do Estado do Pará (UEPA). https://orcid.org/0000-0001-6732-5860

\section{INTRODUÇÃO}

A tualmente, existem inúmeros casos de pessoas infectadas com doenças parasitárias que afetam regulamente a saúde pública da população em geral, estima-se que é a causa de várias enfermidades, principalmente em crianças e idosos, e estima se que, aproximadamente 450 milhões de pessoas no mundo, são infectados diariamente. Está associada a vários fatores, dentre eles estão as instalações sanitárias inadequadas tanto em vias públicas como rurais, fatores socioculturais, a manipulação de alimentos inadequada, e águas contaminadas por parasitas, ausência de saneamento básico e contato com animais contaminados ${ }^{(1)}$.

As hortaliças são transmissoras de parasitoses, pois podem conter cistos de protozoários, ovos e larvas de helminto; pela falta de uma condição sanitária adequada dessas hortaliças, as principais formas de contaminação dessas hortaliças são por contaminação ambiental do solo por uso de adubo com dejetos fecais e poluição fecal da água utilizadas na irrigação dessas hortaliças ${ }^{(2)}$.

Os parasitas são organismos que necessitam do hospedeiro para sobreviver e suas formas parasitárias podem ser helmintos, protozoários ou artrópodes, podem ser de dois tipos: ectoparasitas, que são os parasitas que vivem no ambiente externo; e o endoparasita, os que vivem em ambientes internos ${ }^{(3)}$.

As transmissões desses parasitas, geralmente, ocorrem por via oral passiva, afetando indivíduos de todas as idades, causando anemia, diarreia, perda de peso, diminuição no aprendizado e redução na velocidade de crescimento. A análise da presença de parasitas em hortaliças é muito importante, pois fornece dados das condições de higiene envolvidas na manipulação, manejo, armazenamento, transporte, recipiente e equipamentos contaminados, que podem vir a contaminar os seus consumidores ${ }^{(4)}$.

A maioria dessas doenças ocorre por contaminação principalmente de alimentos, que têm grande importância à saúde da pública, os quais precisam de boas condições higiênica e sanitárias adequadas para o consumo do ser hu$\operatorname{mano}^{(2)}$.

Esses parasitas podem habitar no interior ou exterior do hospedeiro, extraindo alimento e abrigo, que afetam o equilíbrio nutricional do hospedeiro e a absorção de nutrientes, assim, induzindo o sangramento intestinal, redução da ingestão de alimento e até obstrução intestinal ${ }^{(5)}$.
A diarreia, desnutrição, anorexia, emagrecimento e dor abdominal são algumas consequências e sintomas das parasitoses intestinais. Essa doença, muitas vezes, age de forma silenciosa, dificultando o diagnóstico, tratamento adequado e profilaxia de uma possível reinfecção ${ }^{(6)}$.

Dentre as principais parasitoses intestinais, verificam-se: amebíase, giardíase, balantidíase, ascaridíase, ancilostomíase e estrongiloidíase.

A amebíase é causada pelo protozoário Entamoeba Histolytica, que é um pseudópode, que causa proteólise e lise ao tecido, podendo induzir a apoptose da célula do hospedeiro. É transmitida por via fecal-oral, por contaminação de cistos infectantes em alimentos e água, mas também é possível a transmissão sexual, principalmente em práticas oral-anal. Há outras espécies de ameba, mas dentre elas, a Entamoeba Histolytica é a única considerada patogênica. Esse parasita pode manifestar-se de duas formas: intestinal e extra intestinal ${ }^{(7)}$. A forma intestinal apresenta um quadro não patogênico, onde as complicações ocorrem no intestino grosso e quadros patogênicos na parede intestinal, no fígado e em outros órgãos, podendo ocorrer simultaneamente 


\section{artigo}

Lima, B.S.; Aguiar, J.C.; Aguiar, L.M.N.M.; Machado da Silva, D.M.; Carvalho Junior, A.M.; Lima da Silva, L.F.; Matos, R.R.C.;

A presença de parasitas em alfaces (Lactuca Sativa) comercializados em feiras no município de Santarém-PA

Dentre os principais sintomas, a dor abdominal prolongada, a diarreia, a disenteria, a febre, a flatulência e a distensão abdominal. Em casos mais extremos, podem ocorrer necroses extensas da mucosa, colite ulcerativa, anemia, apendicite, peritonite e perfuração intestinal. Já na forma extra intestinal, os trofozoitos chegam nos órgãos através da circulação sanguínea, principalmente no fígado, no qual causam a formação de abscessos e o desenvolvimento de um quadro fatal ${ }^{(7)}$.

A giardíase (Giardia Lamblia) é causada pela Giardia Duodenalis, um parasita do intestino delgado de mamíferos, aves, répteis e anfíbios, que se apresenta de duas formas: trofozoito e cisto. É transmitido pela ingestão de cistos presentes em água e alimentos contaminados ou pela via fecal-oral. É uma parasitose intestinal que afeta o estado físico e nutricional do indivíduo, pois ele se fixa na parede do intestino, impedindo a absorção dos nutrientes. Acometem principalmente crianças, podendo causar retardo no crescimento, por conta da má absorção intestinal. Geralmente, essa parasitose é assintomática, mas quando se tem sintomas, os mais comuns são: diarreias aguda com cólicas intestinais, anorexia, náuseas e vômitos, gases, azia, dentre outros ${ }^{(3)}$.

A balantidíase é causada pelo Balantidium coli, um parasita que tem duas formas: cistos e trofozoitos, é um parasita ciliado. É transmitido através da ingestão de alimentos, água e até mesmo mãos contaminadas. Ele habita, geralmente, na luz do intestino grosso do hospedeiro, não sendo capaz de sozinho penetrar mucosas intactas, só é capaz de penetrar mucosas se já estiver lesada e, como ele produz hialuronidase, aumentando essa lesão inicial até mesmo causar necrose localizadas e úlceras. Os sintomas mais comuns encontrados são: diarreia, meteorismo, anorexia, dor abdominal, fraqueza e febre. Essas lesões e os sintomas são semelhantes ao da amebíase ${ }^{(8)}$.

A ascaridíase é causada pelo nema-
A balantidíase é causada pelo Balantidium coli, um parasita que tem duas formas: cistos e trofozoitos, é um parasita ciliado. É transmitido através da ingestão de alimentos, água e até mesmo mãos contaminadas. Ele habita, geralmente, na luz do intestino grosso do hospedeiro, não sendo capaz de sozinho penetrar mucosas intactas, só é capaz de penetrar mucosas se já estiver lesada e, como ele produz hialuronidase, aumentando essa lesão inicial até mesmo causar necrose localizadas e úlceras. toide Ascaris lumbricoides, popularmente conhecido como lombriga. É o helminto mais frequente em humanos, sendo as crianças as mais afetadas. A forma de infecção é através do ovo embrionado, contendo larvas de segundo estádio e a via de transmissão é oral. A maioria das infecções é leve e clinicamente benigna. Geralmente é assintomática ${ }^{(9)}$.

A ancilostomíase humana pode ser causada por três agentes etiológicos: Ancylostoma duodenalis, Necator americanus e Ancylostoma Ceylanicum. A A. duodenalis e o Necator americanos são os principais ancilostomídeos, em que no estágio adulto desempenham parasitismo gastrintestinal em humanos, já o A. ceylanicum tem como hospedeiro definitivo canídeos e felídeos domésticos, apesar de parasitar humanos também. A A. duodenalis tem sua forma de transmissão via transcutânea e oral, sendo a via oral mais efetiva, e a $N$. americanos, infecta mais por via transcutânea. A A. ceylanicum é a mais disseminada por via oral. Os principais sinais e sintomas são: sensação de picada, hiperemia, prurido, e edema que podem resultar em uma dermatite urticar forme $e^{(10)}$.

A estrongiloidíase é uma infecção causada pelo helminto do gênero Strongyloides. Sua forma de transmissão mais comum é pela penetração da larva através da pele, porém também é transmitido por ingestão de água e alimentos contaminados com larvas de Strongyloide stercoralis. Geralmente, apesar de ter um estímulo do sistema imune, não se consegue eliminar esse parasita, se tornando portador dessa doença de forma crônica. A presença de ovos ou larvas desse helminto na mucosa do intestino delgado pode resultar em inflamações, que podem levar a ulcerações extensas ${ }^{(11)}$.

O consumo de hortaliças se torna muito importante por conta das suas propriedades funcionais, já que a maioria das hortaliças são excelentes fontes de sais minerais, vitaminas e substância 
antioxidante. Porém, o consumo de hortaliças in natura, também podem apresentar riscos à saúde, os principais riscos potenciais estão relacionados às contaminações químicas e microbiológicas que podem ocorrer durante a sua produção $^{(12)}$.

As hortaliças, principalmente, as consumidas cruas, como a alface, são de suma importância para a saúde pública, são muito consumidas pela população, elas têm o risco de conter cisto de protozoários ou ovos e larvas de helmintos, sendo essas, importantes vias de contaminação de parasitas. As principais formas de infecção por parasitas em hortaliças são por águas contaminadas com fezes de origem humana, que se usa na irrigação ou também por contaminação do solo e formas inadequadas de manusear e transportar as hortaliças ${ }^{(13)}$.

A alface (Lactuca sativa L.) da família Asteraceae é uma das hortaliças mais consumida no Brasil, ela é rica em vitaminas $\mathrm{A}, \mathrm{B} 1, \mathrm{~B} 2, \mathrm{~B} 5$, cálcio, potássio, sódio, ferro, silício, flúor, magnésio e também em propriedades medicinais, como: calmante, diurética, péptica, laxante, mineralizaste, desoxidante, vitalizante e depurativa. Além dos seus efeitos benéficos em relação a saúde da população, a alface ela tem seu baixo custo, facilidade para se cultivar, por isso é bastante consumida no Bra$\operatorname{sil}^{(14,15)}$.

Em países em desenvolvimento, nas áreas rurais e urbanas, por conta das baixas condições de saneamento, as parasitoses são frequentes e as hortaliças são uma das principais vias de transmissão de parasitas. $\mathrm{O}$ costume de consumir hortaliças in natura facilita a exposição da maioria da população às formas de infecção por parasitas. Crianças, idosos e pessoas com distúrbios imunes são as que estão mais suscetíveis a ser infectado por esses parasitas oportunistas, como: Giárdia sp, Entamoeba sp, Cryptosporidium sp, e Isospora $\mathrm{sp}^{(4,16)}$.

O presente estudo teve como objetivo analisar a presença de parasitas em alfaces comercializados em feiras da Cidade de Santarém-Pará, as quais essas hortaliças são conhecidas como a hortaliça folhosa mais consumida no Brasil, trazida para o país através dos portugueses em meados do século XVI, a mesma traz benefícios a população sendo bem higienizada até chegar as mesas da população. Diante dessa premissa: Quais os tipos de parasitas encontrados na Lactuca Sativa, nas feiras do município de Santarém-Pará?

\section{METODOLOGIA}

Estudo exploratório, transversal, de método descritivo, e abordagem quanti-qualitativa, realizado em campo e analisado pelo método Hoffman (sedimentação espontânea) a presença de parasitas em alfaces comercializados em feiras do município de Santarém-PA no período de agosto a setembro de 2019.

Foram colhidas, entre os meses de agosto a setembro de 201951 amostras de alfaces (Lactuca sativa) em três feiras de comercialização no município de Santarém-PA. Dentre os ambientes explorados, a Feira da Cohab, a Feira do aeroporto velho e a Feira do Mercadão 2000 , sendo cada uma de bairros dife-

\begin{tabular}{|c|c|c|c|c|c|c|}
\hline Feiras & Balantidium sp. & $\%$ & Giardia sp. & $\%$ & Negativo & $\%$ \\
\hline Aeroporto Velho & 0 & - & 10 & 35,72 & 7 & 43,75 \\
\hline Cohab & 5 & 71,4 & 9 & 32,14 & 3 & 18,75 \\
\hline Mercadão 2000 & 2 & 28,6 & 9 & 32,14 & 6 & 37,5 \\
\hline TOTAL & 7 & 100,0 & 28 & 100,0 & 16 & 100,00 \\
\hline
\end{tabular}

rentes. Em cada feira foram escolhidas 5 bancas distintas e delas foram selecionadas entre 3 a 4 alfaces, totalizando 17 alfaces por feira. Para a obtenção e transporte dessas amostras foram utilizados os sacos plásticos disponibilizadas nas bancas. Após a seleção das hortaliças, foram levadas até o Laboratório Biovida para a realização do processo de análise.

No laboratório, as hortaliças foram desfolhadas em algumas partes e colocadas em um recipiente com água destilada, deixando-a em repouso por 15 minutos. Baseada no método de Hoffman de sedimentação, após os 15 minutos foram retiradas as folhas e o líquido do recipiente foi filtrado através da gaze e deixado em repouso por 24 horas em um cálice cômico, para sedimentação. No dia seguinte, com o auxílio de uma pipeta Pasteur, foi retirado uma quantidade da amostra do sedimento e colocado em uma lâmina com uma gota do corante lugol, e outra lâmina sem o corante lugol e cobrindo-o com a lamínula e analisado em um microscópio óptico pelo método direto. Foram analisados nas objetivas de 40x, a fim de fazer a identificação de estruturas parasitarias, nas 51 amostras de alfaces.

\section{RESULTADOS}

Este estudo foi baseado no método de Hoffman, de sedimentação espontânea, onde foram analisadas 51 amostras, dentre as quais 16 amostras apresentaram-se negativas para alguma estrutura parasita, e 35 amostras apresentaram positividade para estruturas parasitárias, das quais 28 amostras estavam contaminadas com Giardia lamblia e 07 amostras estavam contaminadas com Balantidium coli. Vejamos na Tabela 1 abaixo:

Ao analisar a Tabela 1, observa-se que na feira do Aeroporto Velho tivemos contaminação apenas por Giárdia sp. $(35,72 \%)$ e $43,75 \%$ de amostras negativa para alguma estrutura parasitá- 


\section{artigo}

Lima, B.S.; Aguiar, J.C.; Aguiar, L.M.N.M.; Machado da Silva, D.M.; Carvalho Junior, A.M.; Lima da Silva, L.F.; Matos, R.R.C.;

A presença de parasitas em alfaces (Lactuca Sativa) comercializados em feiras no município de Santarém-PA

ria, comparada a outras duas feiras, foi o local com maior índice de contaminação por Giárdia sp.

$\mathrm{Na}$ feira da Cohab tivemos contaminação por Balantidium sp. $(71,4 \%)$ e Giárdia sp. (32,14\%), sendo mais prevalente a contaminação por Giárdia sp. e $18,75 \%$ de amostras negativas para estrutura parasitária.

$\mathrm{Na}$ feira do Mercadão tivemos também contaminação por Balantidium sp. $(28,6 \%)$ e por Giárdia sp. $(32,14 \%)$ e um total de $37,5 \%$ de amostras negativas para estrutura parasitárias.

Houve prevalência de amostras positivas para alguma estrutura parasitária, sendo a Giárdia sp. o parasita mais predominante com 28 amostras contaminadas, das 51 amostras analisadas. Nas três feiras, a Giárdia sp. foi a mais ocorrente, sendo que na feira do Aeroporto foi a que teve maior índice de contaminação por Giárdia sp.e nenhuma contaminação por Balantidium sp. e a feira da Cohab e Mercadão tiveram a mesma porcentagem de contaminação $(32,14 \%)$ por Giárdia sp. Se fizemos uma comparação a feira da Cohab teve maior índice de contaminação por estrutura parasitárias.

\section{DISCUSSÃO}

Diversas hortaliças, especialmente

Gráfico 1. Porcentagem de amostras de alfaces com ausência ou presença de alguma estrutura parasita segundo os bairros. Santarém-Pará, PA, Brasil, 2019.

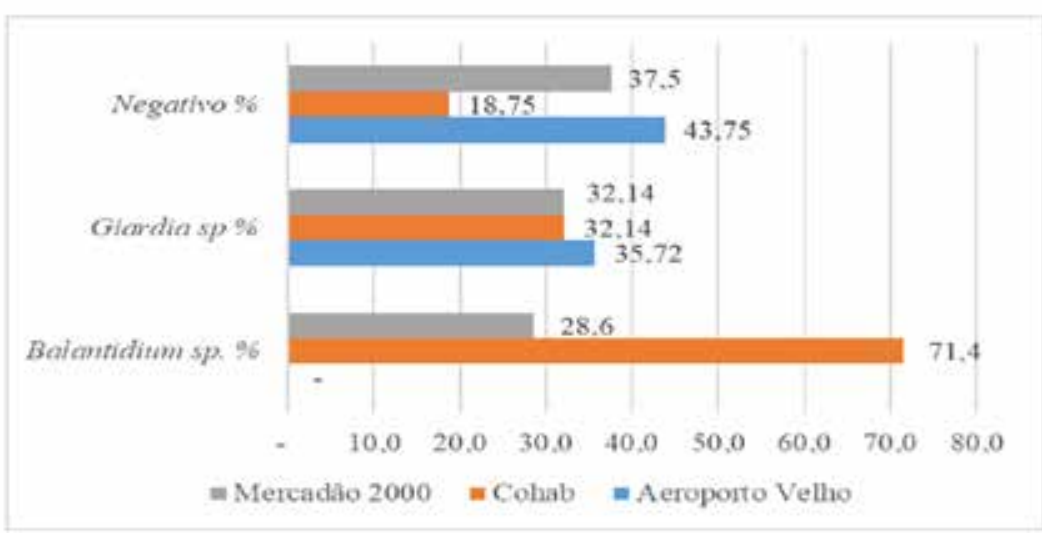

a alface, são frequentemente utilizadas para análises microscópicas com a finalidade de constatar a presença de alguma estrutura parasitária. Os resultados obtidos permitiram avaliar a incidência de contaminação parasitária das amostras de alfaces comercializadas em feiras no município de Santarém-PA, utilizadas neste estudo.

O Gráfico 1 abaixo está representado sobre a incidência para os casos de Giardia sp. e Balantidium sp. encontrados nos locais selecionados por esse estudo; e os resultados negativos também estiveram presentes nas três localidades.

De acordo com o Gráfico 1, dentre os parasitas mais incidentes nas alfaces, observou-se que o Balantidium sp., no bairro da Cohab, sendo bastante ascendente, apresentado 71,4\%, diferente e inferior do identificado no Mercadão (28\%). Em estudos ${ }^{(17)}$ detectou-se, para algum (ns/as) espécie (s) de endoparasita, dentre as 90 amostras estudadas, 63 continham contaminação parasitária, ou seja, $70 \%$.

Ainda no bairro da Cohab, neste Gráfico 1, o índice de Giárdia sp, foi menor e equivalente ao resultado do Mercadão 2000, ambos apresentando $32,14 \%$, diferente do detectado no bairro do Aeroporto Velho (35,72\%), onde a predominância para esse para-

sito fora, ligeiramente, superior aos demais locais, apesar de ser o que mais apresentou negatividade para a contaminação. Em estudo(18) realizado em Niterói-RJ, apesar de não especificados os tipos de parasitas, também constatou-se,que das 120 amostras de alfaces analisadas, 41 estavam contaminadas por algum tipo de parasita, ou seja, 34 $\%$ apresentaram-se de forma positiva.

Em estudo ${ }^{(19)}$, em sua análise a partir de 20 amostras também em feira livre, apontou uma prevalência de Entamoeba histolytica (65\%), Giardia sp. (15\%), Schistosoma mansoni (10\%), Ancylostoma sp. (15\%), Ascaris lumbricoides (5\%) e Trichuris trichiuria (5\%). Por meio deste outro estudo, percebe-se que, as alfaces nem sempre estão isentas por algum tipo habitante do ser que o hospeda.

No Gráfico 1, por conseguinte, em se tratando da negativação dos casos de endoparasita dentro de uma ordem decrescente, bairro do Aeroporto Velho (43,75\%), o Mercadão 2000 $(37,5 \%)$ e a Cohab $(18,75 \%)$, ou seja, novamente, devido ao elevado número de contaminação por Balantidium sp. encontrado no bairro da Cohab, os resultados negativos foram menores em relação aos demais. Vale ressaltar que, dos dois tipos de parasitas identificados, a negativação deve ser considerada, criteriosamente, no Mercadão 2000 e no Aeroporto velho, ao contrário do bairro da Cohab, onde ocorrera menor incidência negativa para um dos tipos desses patógenos.

Segundo a Resolução n. ${ }^{\circ}$ 12/78, da Comissão Nacional de Normas e Padrões para Alimentos (CNNPA), as hortaliças de boa qualidade são aquelas em se encontram com boa qualidade em nível microscópio, sem sujidade, em nível macroscópico e, ainda, isentas de parasitas e/ou larvas. Infelizmente, não só para esse levantamento como também para o de outros autores apresentados, apesar da existência dessa coerente resolução, os problemas de saúde pública relativos a parasitas 
repercutem por falha, especialmente, da manipulação desse vegetal em todas as suas fases, no entanto, gerando insatisfação nos padrões qualitativos e afetando, diretamente aquele que as consomem.

\section{CONCLUSÃO}

A presença desses parasitas em alfaces pode ser um indicativo de má higiene no plantio, irrigação, armazenamento e transporte, já que uma boa higiene é fundamental contra doenças alimentares. O controle e prevenção são possíveis se forem adotadas medidas higiênicas simples, que vão melhorar a qualidade de vida da população.

Conclui-se que os vegetais em questão analisados, os quais foram comercializadas nas feiras do município de

\section{O controle e} prevenção são possíveis se forem adotadas medidas higiênicas simples, que vão melhorar a qualidade de vida da população.
Santarém-PA, podem ter algum destaque fundamental nas parasitoses intestinais, necessitando de medidas profiláticas para melhorar a higiene, tanto o produtor como o consumidor devem tomar essas medidas.

Pretende-se, através desse estudo, elencar embasamento bibliográfico a outras literaturas, assim como na amplitude prática acerca do conhecimento dos parasitas identificados nessas hortaliças; e mecanismo, a nível de visão, especificado sobre os tipos de microrganismos patogênicos à saúde do homem. Sendo assim, a Vigilância Epidemiológica é um órgão proporcionador de ações de detecção e prevenção a qualquer alteração incomum advinda do meio ambiente e capaz de adotar medidas coletivas para a promoção da saúde humana. $\square$

\section{REFERÊNCIAS}

1. Belo VS, et al. Fatores associadoss à ocorrência de parasitoses intestinais em uma populaçâo de crianças e adolescentes. Rev Paul Pediatr. 2012; 30(2):195-201.

2. Silva LP, et al. Avaliação parasitológica em amostras de alfaces( Lactuca sativa var.crispa) comercializadas no município de Quantá, São Paulo, Brazil. Revista Bioscience Journal. 2014; 30(4); 1252-1258.

3. Bener B. O. Parasitologia. São Paulo: Person Education do Brasil; 2015.

4. Quadros RMD, et al. Parasita em alfaces (Lactuca sativa) de mercados e feiras livres de Lages - Santa Catarina. Revista Ciência \& Saúde. 2008; I(2):78-84.

5. Costa-Macêdo LM, et al. Enteroparasitoses em pré-escolares de comunidades favelizadas da cidade do Rio de Janeiro, Brasil. Cad. Saúde Pública. 1998; 14(4):851-855.

6. Melo MCB, et al. Parasitoses intestinais. Revista Médica de Minas Gerais. 2004; 3-12.

7. Rocha A. Parasitologia. São Paulo: RIDEEL; 2013.

8. Rey L. Parasitologia: parasitos e doenças parasitárias do homem nos trópicos ocidentais. 4. ed. Rio de Janeiro: Guanabara Koogan; 2013.

9. Neves DP. Parasitologia Humana. 11. ed. São Paulo: ATHENEU; 2005.

10. Neves DP. Parasitologia Humana. 12. ed. São Paulo: ATHENEU; 2011.

11. Cirmenman SC. Parasitologia Humana e Seus Fundamentos Gerais. 2. ed. São Paulo: Atheneu; 2010.
12. Mattos LM, et al. Produção segura e rastreabilidade de hortaliças. Hortic. Bras. 2009; 27(4):408-413.

13. Soares B, Cantos GA. Detecção de estruturas parasitárias em hortaliças comercializadas na cidade de Florianópolis, SC, Brasil. Rev. Bras. Cienc. Farm. 2006; 42(3):455-460.

14. Carminate $B$, et al. Levantamento de Enteroparasitas em hortaliças comercializadas no município de Pedro Canário, ES, Brasil. Enciclopédia Biosfera. 2011; 7(12):1-7.

15. Fernandes NDS, et al. Avaliação Parasitológica de Hortaliças: da horta ao consumidor final. Saúde e Pesquisa, Maringá. 2015; 8(2):255-265.

16. Mesquita VCL, Serra CMB, Bastos OMP, Uchoa CMA. Contaminação por enteroparasitas em hortaliças comercializadas nas cidades de Niterói e Rio de Janeiro, Brasil. Rev. Soc. Bras. Med. Trop. 1999; 32(4):363-366.

17. Alves ADS, et al. Parasitos em alface-crespa (Lactuca sativaL.), de plantio convencional, comercializada em supermercados de Cuiabá, Mato Grosso, Brasil. Revista de Patologia Tropical. 2013 abr.-jun.; 42(2):217-229.

18. FRANÇA BR, et al. Qualidade Higiênico Sanitária de Alfaces (Lactuca sativa) Comercializados em Feiras Livres na Cidade de Uberlândia,MG,Brasil. Bioscience Journal. 2014 jun; 30(1):458466.

19. Silva VGD, et al. Enteroparasitas Veiculados em Folhas de Alfaces(Lactuca sativa) Comercializados na Feira Livre da Cidade de Governador Valadares, Minas Gerais. Eciclopédia Biosfera. 2017; 14(25):1343-1352. 